\title{
The Cognitive Turn of Translation Study in Artificial Intelligence Era
}

\author{
Rui Ma \\ Xi'an Polytechnic University, Humanities and Social Science Department, \\ Golden Flower South Road 19\#, Xi'an, China \\ marui@xpu.edu.cn
}

Keywords: Translation, Cognition, Artificial Intelligence

\begin{abstract}
Under the initiative of building a Silk Road Economic Belt and 21st Century Maritime Silk Road, China encounters more opportunities in this Artificial Intelligence Era. Translation as a multi-subjects discipline needs to find its own way to survive. The author of the present paper believes that due to its cognitive nature, translation should make more efforts to its cognitive interface which will promote the development of Artificial Intelligence.
\end{abstract}

\section{Introduction}

On December the $28^{\text {th }} 2009$, Prof. Cai Shushan from Tsinghua University gave a lecture under the name of "Explore the Secret of Human Cognition". During this lecture, he mentioned that in $21^{\text {st }}$ century, there are two secrets concerning human beings will be unveiled: one will be genetic science which aims at discovering the secret of life; the other will be cognitive science which hopes to find out the black box of human cognitive ability (Cai Shushan, 2009). He compared gene and cognitive ability to two microcircuits that record our life information and cognition information respectively. If one can mix these two microcircuits together, he will make an artificial man (ibid). Almost 10 years later, Prof. Cai's prophecy comes true. Google released an AI tool that makes sense of our genome. AI tool named Deep Variant could help us turn information gleaned from genetic sequencing into life-saving therapies (Will Knight, 2017). This is an excellent example which shows the combination of genome and cognition science and also fantasized our future. Under such circumstances, several serious questions concerned arise. First, can machine translation totally replace the redundant and laboring human work? Second, how can translation transform in this New Era?

\section{The Market Need and New Opportunity}

In September and October 2013, Chinese President Xi Jinping proposed to build a Silk Road Economic Belt and 21st Century Maritime Silk Road Initiative during his visit to central and Southeast Asia. This initiative was later been formulated into a national developing strategy and encouraged by government from all social aspects. The essence of this idea is to enhance cooperation with countries along the "Silk Rroad" which covers European countries, Asia as well as African countries (Wang Yizhi, 2015). The whole picture can be seen in fig.1.

As a result, the translation market is huge. According to the China language Service Development report 2012, the annual output value of language service enterprises will exceed 260 billion yuan by 2015. In the next decade, the number of languages to be translated will increase from the current 7 source languages and 60 target languages to 200 source languages and 200 target languages. (TAUS, 2013) 


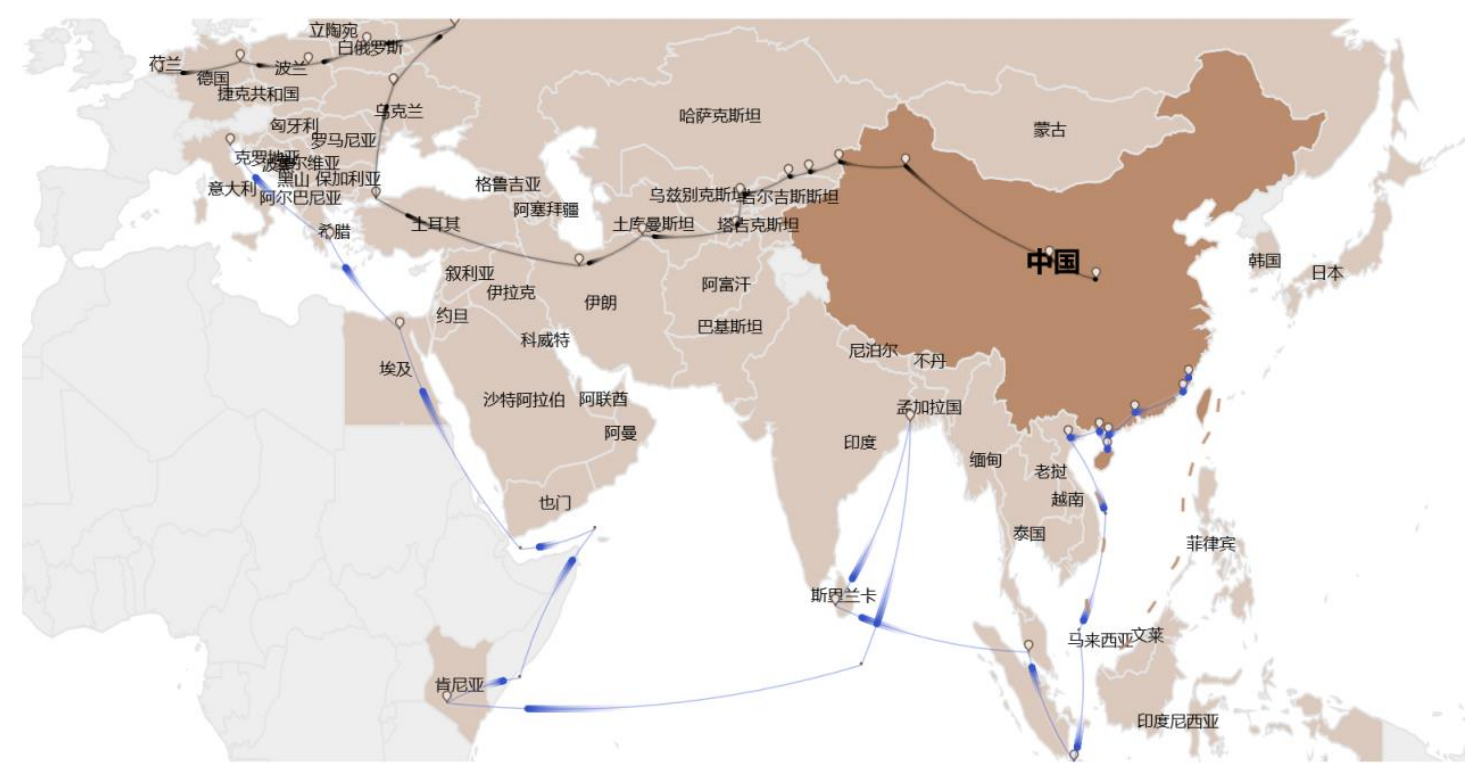

Fig.1 (Cited from http://www.ydylcn.com/skwx_ydyl/sublibrary?SiteID=1\&ID=8721)

\section{Cognitive Turn of Translation}

\subsection{New challenges}

Although the translation demand is huge, it also ushers new challenges. Because the present machine translation cannot completely meet the needs of the market. At present, there are at least 14 translation tools on the internet. For instance, google translation, bing translation, yeekit translation, youdao translation, iciba etc. Although they use different algorithm, no translation tool is perfect. When we conduct some complex sentences, the translations cannot fully "understand" it. Thus, there is an urgent need for us to further develop these translation tools. One of the core development mechanism behind these translation tools is the help of cognitive science. Also due to this reason, translation itself is also called a multi-subject science. As a matter of fact, translation is a cross lingual and cross - cultural linguistic practice. The process of translation is the process of translating the text from the original text and the original readers. This process is restricted by both the primitive culture and the text symbol, which is also known as the implicit text (Wang Keyou, 2008).

\subsection{Cognitive nature}

The cognitive nature of translation comes a long way over the past years. At first it is considered "as an analytical process", the root of which lies in the "form-content" view of language and meaning. This view regards language as container / vessel, and meaning as content wrapped in a language shell, which needs to be broken to remove the content (215)( Reddy, Michael. 1979).

One of its consequences is that meaning is regarded as a substance attached to words, which can be packed or disassembled, can be accommodated, transferred, packed and lost. Meaning, like the liquid in a container, belongs to the container, so each word has its own meaning. This gives rise to the idea of meaning internalism, on the basis of which translation can only be understood as finding a container of words with the same or similar content in another language: identifying the content by the container is called decoding. Finding a matching container through content is called coding (216).

As a matter of fact, the author believes that this theory is helpful to machine translation, where the text of machine translation is mostly confined to specialized fields such as science and technology. Language in these fields requires the unity of the meaning of words and the unity of format. Vocabulary and meaning basically satisfy one-to-one fixed correspondence, so the language 
is similar to signal under such circumstances.

But this theory lacks sufficient explanation for natural language translation. Meaning is formed in the long-term interaction between language and experience. The meaning of language goes into long-term memory with language acquisition. The brain stores the relationship between our experience and linguistic symbols into long-term memory, which activates each other. When one sees a symbol, one evokes experience, and when one encounters experience, one associates with the corresponding symbol. To a certain extent, people's response to linguistic meaning is automatic and therefore subconscious. This automatism creates the illusion that meaning comes from within the symbol (132). In essence, any sorts of understanding is a kind of "dialogue", including reading. Dialogue embodies the intertextuality in the process of communication. It is a silent dialogue between the author and the reader. On the other hand, if we regard the work as text, and the knowledge, emotion by which the author reacts as the internal text, the communication between the reader and the text can be called intertextuality. From this perspective, understanding is the integration of the tangible language text of the work and the intangible knowledge text of the reader (77). Such kind of job definitely requires more complicated algorithm and cross subject techniques.

To what extent the text of translation can fully express the original work depends on the understanding of reader as well as translator's ability to reproduce it. Therefore, we could see the process of translation as a destination that can never be reached (26). Translation is a process of re-creation after the process of understanding: symbolizing the subjective world and objective world of the author. However, from the original work of the author there is no available and ready access to the author's world, nor is it possible to obtain an accurate objective world (238). Therefore, it need human intelligence to distinguish and analyze it.

\section{Summary}

From the above analysis, we can see that, translation as a career may encounter some hardships. Some of the primitive jobs may disappear as translation machines replace them. But due to the cognitive nature of translation, in this Artificial Intelligence Era, the author believes translation will play a more important role on this higher platform.

\section{References}

[1] Cai Shushan. Exploration of the Human mind: on Cognitive Science. (2009). Zhejiang Humanities Hall. Reported on Nov.28 ${ }^{\text {th }}$. Retrieved on April 19th 2018 <http://culture.zjol.com.cn/05culture/system/2009/12/04/016126265.shtml>

[2] Will Knight, Google Has Released an AI Tool That Makes Sense of Your Genome, December 4, 2017. Retrieved on April 20th, 2018. https://www.technologyreview.com/s/609647/google-has-released-an-ai-tool-that-makes-senseof-your-genome/

[3] Wang Yizhi. An Analysis of China-EU Cooperation on the Maritime Silk Road. International Development Cooperation (2):6-13, 2015.

[4] Jaap van der Meer,Choose Your Own Translation Future. TAUS, 2013.

[5] Corpus of : The Belt and $<$ http://www.ydylcn.com/skwx_ydyl/sublibrary?SiteID=1\&ID=8721>

[6] Wang Keyou. Translation as Process and as Product: A Perspective of Cognition, Language Communication and Meaning. (2008).China Social Sciences Press: 215, 216, 132, 77, 26, 238, 346.

[7] Reddy, Michael, The Conduit Metaphor: a Case of Frame Conflict in our Language about 
Language, in A. Ortony, ed., Metaphor and Thought. Cambridge: Cambridge University Press, p284-310, 1979.

[8] Shen Jingju. Illusions and misunderstandings in Translation. Collected on Theoretical Construction and Cultural Perspective of Translation.(2000).ShanghaiForeign Language Education Press

[9] Tan Yesheng, Prolegomena to Cognitive Translatology: Cognitive Paths and Constraints of Creative Translation, Shang Hai Foreign Education Press, 2010 